\title{
Stability of the bicriteria Boolean investment problem subject to extreme optimism and pessimism criteria
}

\author{
Vladimir Korotkov ${ }^{1, *}$, Yury Nikulin ${ }^{1}$ and Vladimir Emelichev ${ }^{2}$ \\ ${ }^{1}$ Department of Mathematics and Statistics, University of Turku \\ Assistentinkatu 7, 20014 Turku, Finland \\ E-mail: 〈\{vlakor,yurnik\}@utu.fi〉 \\ ${ }^{2}$ Mechanics and Mathematics Faculty, Belarusian State University \\ Nezavisimosty 4, 220030 Minsk, Belarus \\ E-mail: 〈emelichev@bsu.by,emelichev@tut.by〉
}

\begin{abstract}
We consider the bicriteria investment Boolean problem of finding the Pareto set based on efficiency and risk criteria. The quantitative stability characteristics of the problem are investigated, and lower and upper bounds for a stability radius are obtained for the case where portfolio and financial market state spaces are endowed with the Hölder metric. Calculation of these bounds provides investors with a deeper insight into the specific problem of facilitating financial decisions more reliably in uncertain environments.
\end{abstract}

Key words: bicriteria, investment portfolio, portfolio risk, Pareto set, stability radius, Hölder metric

Received: July 4, 2014; accepted: March 3, 2015; available online: March 30, 2015

DOI: 10.17535 /crorr. 2015.0016

\section{Introduction}

Investors face risk challenges when investing in high-yield assets. The main objective in capital management is finding a trade-off between risk and expected return. Based on the Markowitz portfolio theory [12, 14], risk can be reduced through investment diversification in various assets and portfolio selection subject to required income level and acceptable risk. Applying the portfolio theory enables formulating the problem of selecting a set of Pareto optimal portfolios with maximum efficiency for a favorable situation or for a worst-case situation with the lowest risk. The initial data for this problem is obtained through statistical and expert evaluations of risk and efficiency [5]. The initial data can be specifically adjusted through post-optimal analysis in order to determine quantitative stability indicators of solutions [1,11]. One such

\footnotetext{
${ }^{*}$ Corresponding author.
} 
characteristic is the stability radius of the problem, indicating the extent to which the initial data can change without the threat of a new Pareto optimal portfolio appearing. The concept of stability radius has been extensively presented and analyzed in recent literature, focusing on formulating analytical expressions and bounds (see e.g. $[3,6,13,17]$ ). The stability radius depends on the selected metric in the parameter space of the problem. Different metrics take into account differently parameter perturbations of the problem. For example, in the case of the Chebyshev metric $\boldsymbol{l}_{\infty}$ we consider only the maximum absolute value of changes in the initial data, causing perturbations to be more independent. Alternatively, in the case of the Manhattan metric $l_{1}$, we monitor every change. The Hölder metric $l_{p}, 1 \leq p \leq \infty$, includes the Chebyshev and the Manhattan metrics as extreme cases, and also the Euclidean metric. Thus, the Hölder metric enables us to vary control of perturbations in the initial data.

This paper explains how we obtain the lower and upper bounds for the stability radius of a bicriteria investment portfolio problem based on conflicting criteria, such as the extreme optimism criterion for a yield and the extreme pessimism criterion for a risk of lost profit. For the stability analysis of the investment problem relating to perturbations in the initial data (evaluations of investment project efficiency and risk measure), we suppose that the arbitrary Hölder metric is given in the space of portfolios and the space of financial market states.

Earlier in the literature, similar results were obtained in $[7,8,9,10]$ only for particular cases upon determining the Manhattan $l_{1}$ and Chebyshev $\boldsymbol{l}_{\infty}$ metrics in a three-dimensional space of the problem parameters,

\section{Problem statement and basic definitions}

Based on the Markowitz investment theory, we consider a bicriteria variant of a Boolean investment management problem. Let $n$ be the number of alternative investment projects (assets), $m$ the number of predictive states (situations) of the financial market, i.e. the number of variant market scenarios. Then let $x_{j}=1$ if the $j$-th project is chosen, $j \in N_{n}=\{1,2, \ldots, n\}$, or $x_{j}=0$ otherwise. The Boolean vector $x=\left(x_{1}, x_{2}, \ldots, x_{n}\right)^{T}$ is an investment portfolio, and $X \subset \mathrm{E}^{n}, \mathrm{E}=\{0,1\}$, is the set of all investment portfolios, i.e. those realizations not exceeding the budget.

For each market state $i \in N_{m}$, the investment portfolio $x \in X$ is evaluated using an index of efficiency and risk (additive functions):

$$
\sum_{j \in N_{n}} e_{i j} x_{j} \text { and } \sum_{j \in N_{n}} r_{i j} x_{j} .
$$


optimism and pessimism criteria

Here $e_{i j}$ is the expected evaluated efficiency of the project $j \in N_{n}$ for the case when the market is in the state $i \in N_{m}=\{1,2, \ldots, m\} ; r_{i j}$ is the risk measure, which an investor may encounter if choosing the $j$-th project in the $i$-th market state.

Note that there are several approaches to evaluating the efficiency of investment projects (see e.g. $[2,4,15,18]$ ). The initial data are given by two matrices - the efficiency matrix $E=\left[e_{i j}\right] \in \mathbb{R}^{m \times n}$ and the risk matrix $R=\left[r_{i j}\right] \in \mathbb{R}^{m \times n}$. The investor wants to select the most profitable portfolio, which always conflicts with desiring the lowest investment risk level. These circumstances, together with market uncertainty and lack of information on selection conditions for projects, require the use of two well- known extreme optimism and extreme pessimism criteria.

$$
\begin{aligned}
\operatorname{MAXMAX} e(x, E) & =\max _{i \in N_{m}} e_{i} x \\
& =\max _{i \in N_{m}} \sum_{j \in N_{n}} e_{i j} x_{j} \rightarrow \max _{x \in X}, \\
\text { MINMAX } r(x, R) & =\max _{i \in N_{m}} r_{i} x \\
& =\max _{i \in N_{m}} \sum_{j \in N_{n}} r_{i j} x_{j} \rightarrow \min _{x \in X},
\end{aligned}
$$

where $e_{i}=\left(e_{i 1}, e_{i 2}, \ldots, e_{\text {in }}\right), r_{i}=\left(r_{i 1}, r_{i 2}, \ldots, r_{\text {in }}\right)$ are the $i$-th rows of matrices $E$ and $R$ respectively. Using the first (MAXMAX) criterion, a venturesome investor optimizes the portfolio efficiency $e_{i} x$ when judging that the market is in its most favorable state, i.e. maximal portfolio efficiency. It is worth noting that these situations requiring certain behavior are quite common in economics. Such attitudes are inherent not only in optimistic investors but also in those who are cornered in particular situations. The other bottleneck (MINMAX) criterion is usually called the Savage criterion [16].

The bicriteria Boolean problem relating to project selection $Z(E, R)$ requires searching the Pareto set (or the set of Pareto optimal portfolios)

$$
P(E, R)=\{x \in X: P(x, E, R)=\varnothing\},
$$

where

$P(x, E, R)=\left\{x^{\prime} \in X: e(x, E) \leq e\left(x^{\prime}, E\right) \& r(x, R) \geq r\left(x^{\prime}, R\right) \& f(x, E, R) \neq f\left(x^{\prime}, E, R\right)\right\}$.

Here $f(x, E, R)=(e(x, E), r(x, R))$.

The Pareto set comprises portfolios, for which it is impossible to make any criterion can be improved without worsening the other criterion of the selected portfolio. In our case, a portfolio is Pareto optimal when expressing optimal trade-off between risk and effectiveness. 
In the portfolio space $\mathbb{R}^{n}$ and the financial market state space $\mathbb{R}^{m}$, we introduce the Hölder metric $l_{p}, 1 \leq p \leq \infty$, i.e. for any matrix $D=\left[d_{i j}\right] \in \mathbb{R}^{m \times n}$ with rows $d_{i} \in \mathbb{R}^{n}, i \in N_{m}$, we attain equality

where

$$
\|D\|_{p}=\left\|\left(\left\|d_{1}\right\|_{p},\left\|d_{2}\right\|_{p}, \ldots,\left\|d_{m}\right\|_{p}\right)\right\|_{p},
$$

It is easy to see that

$$
\left\|d_{i}\right\|_{p}= \begin{cases}\left(\sum_{j \in N_{n}}\left|d_{i j}\right|^{p}\right)^{1 / p}, & \text { if } 1 \leq p<\infty, \\ \max \left\{\left|d_{i j}\right|: j \in N_{n}\right\}, & \text { if } p=\infty .\end{cases}
$$

$$
\left\|d_{i}\right\|_{p} \leq\|D\|_{p}, \quad i \in N_{m}, p \in[1, \infty] .
$$

Besides the number $p$, we also use the dual number $q$, as defined by the equality expression

$$
\frac{1}{p}+\frac{1}{q}=1
$$

where $q=1$ if $p=\infty$, and $q=\infty$ if $p=1$. Thus, later, we assume that the variation domain of $p$ and $q$ is the interval $[1, \infty]$, with $p, q$ fulfilling the above conditions, and we assume $1 / p=0$ for $p=\infty$.

Using the well-known Hölder's inequality

$$
a b \leq\|a\|_{p}\|b\|_{q},
$$

where $a=\left(a_{1}, a_{2}, \ldots, a_{n}\right) \in \mathbb{R}^{n}, \quad b=\left(b_{1}, b_{2}, \ldots, b_{n}\right)^{T} \in \mathbb{R}^{n}, \quad$ it is easy to see that for $x, x^{\prime} \in X$ and $i, i^{\prime} \in N_{m}$ the following inequalities

$$
d_{i} x-d_{i^{\prime}} x^{\prime} \geq-\|D\|_{p}\left\|\left(\|x\|_{q},\left\|x^{\prime}\right\|_{q}\right)\right\|_{q}
$$

hold for any matrix $D \in \mathbb{R}^{m \times n}$. Indeed, if $i=i^{\prime}$, then

If $i \neq i^{\prime}$, then

$$
\begin{aligned}
d_{i} x-d_{i^{\prime}} x^{\prime} & \geq-\left\|d_{i}\right\|_{p}\left\|x-x^{\prime}\right\|_{q} \\
& \geq-\|D\|_{p}\left\|x-x^{\prime}\right\|_{q}=-\|D\|_{p}\left(\sum_{j \in N_{n}}\left(x_{j}-x_{j}^{\prime}\right)\right)^{1 / q} \\
& \geq-\|D\|_{p}\left(\sum_{j \in N_{n}}\left(x_{j}+x_{j}^{\prime}\right)\right)^{1 / q}=-\|D\|_{p}\left\|\left(\|x\|_{q^{\prime}},\left\|x^{\prime}\right\|_{q}\right)\right\|_{q} .
\end{aligned}
$$

$$
\begin{aligned}
d_{i} x-d_{i^{\prime}} x^{\prime} & \geq-\left\|d_{i}\right\|_{p}\|x\|_{q}-\left\|d_{i^{\prime}}\right\|_{p}\left\|x^{\prime}\right\|_{q} \\
& \geq-\left\|\left(\left\|d_{i}\right\|_{p^{\prime}},\left\|d_{i^{\prime}}\right\|_{p}\right)\right\|\left\|_{p}\right\|\left(\|x\|_{q^{\prime}},\left\|x^{\prime}\right\|_{q}\right) \|_{q} \\
& \geq-\|D\|_{p}\left\|\left(\|x\|_{q},\left\|x^{\prime}\right\|_{q}\right)\right\|_{q} .
\end{aligned}
$$

Similar to $[7,9,10]$, the stability radius of the investment problem $Z(E, R)$ is defined as: 
where

$$
\rho=\rho_{p}^{m}(E, R)= \begin{cases}\sup \Xi & \text { if } \Xi \neq \varnothing, \\ 0 & \text { if } \Xi=\varnothing,\end{cases}
$$

$$
\Xi=\left\{\varepsilon>0: \forall\left(E^{\prime}, R^{\prime}\right) \in \Omega(\varepsilon) \quad\left(P\left(E+E^{\prime}, R+R^{\prime}\right) \subseteq P(E, R)\right)\right\},
$$

$\Omega(\varepsilon)=\left\{\left(E^{\prime}, R^{\prime}\right) \in \mathbb{R}^{m \times n} \times \mathbb{R}^{m \times n}: \max \left\{\left\|E^{\prime}\right\|_{p^{\prime}},\left\|R^{\prime}\right\|_{p}\right\}<\varepsilon\right\}$ is the set of perturbing matrices, $P\left(E+E^{\prime}, R+R^{\prime}\right)$ is the Pareto set of the perturbed problem $Z\left(E+E^{\prime}, R+R^{\prime}\right)$.

Obviously, if the equality $P(E, R)=X$ holds, the stability radius of the problem $Z(E, R)$ is equal to infinity. Therefore, this case will be excluded from our consideration. The problem $Z(E, R)$, for which the set $X \backslash P(E, R)$ is nonempty, is called a nontrivial one.

\section{Stability radius bounds}

For $x, x^{\prime} \in X$ we have the following

$$
\begin{aligned}
\gamma\left(x, x^{\prime}\right) & =\min \left\{e\left(x^{\prime}, E\right)-e(x, E), r(x, R)-r\left(x^{\prime}, R\right)\right\}, \\
\varphi & =\varphi_{p}^{m}(E, R)=\min _{x \notin P(E, R)} \max _{x^{\prime} \in P(x, E, R)} \frac{\gamma\left(x, x^{\prime}\right)}{\left\|\left(\|x\|_{q},\left\|x^{\prime}\right\|_{q}\right)\right\|_{q}}, \\
\psi & =\psi^{m}(E, R)=\min _{x \notin P(E, R)} \max _{x^{\prime} \in P(x, E, R)} \frac{\gamma\left(x, x^{\prime}\right)}{\left\|x-x^{\prime}\right\|_{1}} .
\end{aligned}
$$

It is clear that $\varphi$ and $\psi$ are nonnegative numbers.

Theorem 1. For $m \in \mathbb{N}$ and $p \in[1, \infty]$, the stability radius $\rho_{p}^{m}(E, R)$ has the following lower and upper bounds

$$
\varphi_{p}^{m}(E, R) \leq \rho_{p}^{m}(E, R) \leq(m n)^{1 / p} \psi^{m}(E, R) .
$$

Proof. First, we prove that $\rho \geq \varphi$. It is evident that $\varphi=0$. Let $\varphi>0$. According to the definition of $\varphi$, for any portfolio $x \notin P(E, R)$ there exists a portfolio $x^{0} \in P(x, E, R)$ such that

i.e. the inequalities

$$
\gamma\left(x, x^{0}\right) \geq \varphi\left\|\left(\|x\|_{q},\left\|x^{0}\right\|_{q}\right)\right\|_{q},
$$

$$
\begin{aligned}
& e\left(x^{0}, E\right)-e(x, E) \geq \varphi\left\|\left(\|x\|_{q},\left\|x^{0}\right\|_{q}\right)\right\|_{q}, \\
& r(x, R)-r\left(x^{0}, R\right) \geq \varphi\left\|\left(\|x\|_{q},\left\|x^{0}\right\|_{q}\right)\right\|_{q}
\end{aligned}
$$

hold. 
Hence, using (1), for any pair of matrices $\left(E^{\prime}, R^{\prime}\right) \in \Omega(\varphi)$ with the rows $e_{i}^{\prime} \in \mathbb{R}^{n}$ and $r_{i}^{\prime} \in \mathbb{R}^{n}, i \in N_{m}$, we have

and

$$
\begin{aligned}
e\left(x^{0}, E+E^{\prime}\right)-e\left(x, E+E^{\prime}\right) & =\max _{i \in N_{m}}\left(e_{i}+e_{i}^{\prime}\right) x^{0}-\max _{i \in N_{m}}\left(e_{i}+e_{i}^{\prime}\right) x \\
& =\min _{i \in N_{m}} \max _{i^{\prime} \in N_{m}}\left(e_{i^{\prime}} x^{0}+e_{i^{\prime}}^{\prime} x^{0}-e_{i} x-e_{i}^{\prime} x\right) \\
& \geq e\left(x^{0}, E\right)-e(x, E)-\left\|E^{\prime}\right\|_{p}\left\|\left(\|x\|_{q^{\prime}},\left\|x^{0}\right\|_{q}\right)\right\|_{q} \\
& \geq\left(\varphi-\left\|E^{\prime}\right\|_{p}\right)\left\|\left(\|x\|_{q^{\prime}},\left\|x^{0}\right\|_{q}\right)\right\|_{q}>0,
\end{aligned}
$$

$$
\begin{aligned}
r\left(x, R+R^{\prime}\right)-r\left(x^{0}, R+R^{\prime}\right) & =\max _{i \in N_{m}}\left(r_{i}+r_{i}^{\prime}\right) x-\max _{i \in N_{m}}\left(r_{i}+r_{i}^{\prime}\right) x^{0} \\
& =\min _{i^{\prime} \in N_{m}} \max _{i \in N_{m}}\left(r_{i} x+r_{i}^{\prime} x-r_{i^{\prime}} x^{0}-r_{i^{\prime}}^{\prime} x^{0}\right) \\
& \geq r(x, R)-r\left(x^{0}, R\right)-\left\|R^{\prime}\right\|_{p}\left\|\left(\|x\|_{q^{\prime}},\left\|x^{0}\right\|_{q}\right)\right\|_{q} \\
& \geq\left(\varphi-\left\|R^{\prime}\right\|_{p}\right)\left\|\left(\|x\|_{q},\left\|x^{0}\right\|_{q}\right)\right\|_{q}>0 .
\end{aligned}
$$

i.e. the portfolio $x$ is not Pareto optimal in the perturbed problem $Z\left(E+E^{\prime}, R+R^{\prime}\right)$. In summarizing and considering $x \notin P(E, R)$, we conclude that

$$
\forall\left(E^{\prime}, R^{\prime}\right) \in \Omega(\varphi) \quad\left(P\left(E+E^{\prime}, R+R^{\prime}\right) \subseteq P(E, R)\right) .
$$

Therefore, the inequality $\rho \geq \varphi$ is true.

Next, we prove the inequality $\rho \leq(m n)^{1 / \rho} \psi$. According to the definition of $\psi$, there exists a portfolio $x^{*} \notin P(E, R)$ such that for any portfolio $x \in P\left(x^{*}, E, R\right)$ one of the following inequalities is true:

$$
\begin{aligned}
& e(x, E)-e\left(x^{*}, E\right) \leq \psi\left\|x-x^{*}\right\|_{1}, \\
& r\left(x^{*}, R\right)-r(x, R) \leq \psi\left\|x-x^{*}\right\|_{1} .
\end{aligned}
$$

We consider the first variant. Let inequality (2) hold. Setting $\varepsilon>(m n)^{1 / p} \psi$, we define the elements of the matrices $E^{0}=\left[e_{i j}^{0}\right] \in \mathbb{R}^{m \times n}$ and $R^{0}=\left[r_{i j}^{0}\right] \in \mathbb{R}^{m \times n}$ with rows $e_{i}^{0}$ and $r_{i}^{0}, i \in N_{m}$ as follows:

$$
\begin{aligned}
& e_{i j}^{0}= \begin{cases}\delta & \text { if } i \in N_{m}, x_{j}^{*}=1, \\
-\delta & \text { otherwise, }\end{cases} \\
& r_{i j}^{0}=0, \quad(i, j) \in N_{m} \times N_{n},
\end{aligned}
$$

where $\varepsilon /(m n)^{1 / p}>\delta>\psi$. Then we have

$$
\left\|e_{i}^{0}\right\|_{p}=n^{1 / p} \delta, \quad i \in N_{m},\left\|E^{0}\right\|_{p}=(m n)^{1 / p} \delta,\left\|R^{0}\right\|_{p}=0 .
$$


Therefore, $\left(E^{0}, R^{0}\right) \in \Omega(\varepsilon)$. In addition, all the rows $e_{i}^{0}, i \in N_{m}$, of the matrix $E^{0}$ are the same and consist of the components $\delta$ and $-\delta$. Denoting $A=e_{i}^{0}, i \in N_{m}$, we get $\|A\|_{p}=n^{1 / p} \delta$,

$$
A\left(x-x^{*}\right)=-\delta\left\|x-x^{*}\right\|_{1}<0 .
$$

Hence, taking into account (3), we deduce that for any portfolio $x \in P\left(x^{*}, E, R\right)$

As a result, we obtain

$$
\begin{aligned}
e\left(x, E+E^{0}\right)-e\left(x^{*}, E+E^{0}\right) & =\max _{i \in N_{m}}\left(e_{i}+e_{i}^{0}\right) x-\max _{i \in N_{m}}\left(e_{i}+e_{i}^{0}\right) x^{*} \\
& =\min _{i \in N_{m}} \max _{i^{\prime} \in N_{m}}\left(e_{i^{\prime}} x+e_{i^{\prime}}^{0} x-e_{i} x^{*}-e_{i}^{0} x^{*}\right) \\
& \leq \min _{i \in N_{m}} \max _{i^{\prime} \in N_{m}}\left(e_{i^{\prime}} x-e_{i} x^{*}\right)+A\left(x-x^{*}\right) \\
& =e(x, E)-e\left(x^{*}, E\right)+A\left(x-x^{*}\right) \\
& \leq(\psi-\delta)\left\|x-x^{0}\right\|_{1}<0 .
\end{aligned}
$$

$$
\forall x \in P\left(x^{*}, E, R\right)\left(x \notin P\left(x^{*}, E+E^{0}, R+R^{0}\right)\right) .
$$

Now, let the portfolio be $x \notin P\left(x^{*}, E, R\right)$. Then the following three cases are possible.

Case 1. $f(x, E, R)=f\left(x^{*}, E, R\right)$, i.e.

$$
\begin{aligned}
& e(x, E)=e\left(x^{*}, E\right), \\
& r(x, R)=r\left(x^{*}, R\right) .
\end{aligned}
$$

Then, using (5), we have

$$
\begin{aligned}
e\left(x, E+E^{0}\right)-e\left(x^{*}, E+E^{0}\right) & =\max _{i \in N_{m}}\left(e_{i}+e_{i}^{0}\right) x-\max _{i \in N_{m}}\left(e_{i}+e_{i}^{0}\right) x^{*} \\
& =e(x, E)-e\left(x^{*}, E\right)+A\left(x-x^{*}\right)<0 .
\end{aligned}
$$

Case 2. $e(x, E)<e\left(x^{*}, E\right)$. Then, using (5) again, we deduce that

$$
e\left(x, E+E^{0}\right)-e\left(x^{*}, E+E^{0}\right)=e(x, E)-e\left(x^{*}, E\right)+A\left(x-x^{*}\right)<0 .
$$

Case 3. $r(x, R)>r\left(x^{*}, R\right)$. Then, considering the structure of the matrix $R^{0}$ , we get

$$
r\left(x, R+R^{0}\right)>r\left(x^{*}, R+R^{0}\right) .
$$

Thereby, $x \notin P\left(x^{*}, E+E^{0}, R+R^{0}\right)$, if $x \notin P\left(x^{*}, E, R\right)$. Combining with (6), we obtain $P\left(x^{*}, E+E^{0}, R+R^{0}\right)=\varnothing$, i.e. $x^{*} \in P\left(E+E^{0}, R+R^{0}\right)$. Hence, in connection with $x^{*} \notin P(E, R)$, we conclude that for (3) the following formula is true:

$$
\forall \varepsilon>(m n)^{1 / p} \psi \quad \exists\left(E^{0}, R^{0}\right) \in \Omega(\varepsilon): \quad\left(P\left(E+E^{0}, R+R^{0}\right) \nsubseteq P(E, R)\right) .
$$


The same formula has been obtained, providing similar proof in the second variant, that is, for (4). Hence, $\rho \leq(m n)^{1 / p} \psi$. Theorem 1 has been proven.

\section{Corollaries and examples}

Theorem 1 implies several corollaries.

Corollary 1. For $m \in \mathbb{N}$, the stability radius $\rho_{\infty}^{m}(E, R)$ has the following lower and upper bounds

$$
\min _{x \notin P(E, R)} \max _{x^{\prime} \in P(x, E, R)} \frac{\gamma\left(x, x^{\prime}\right)}{\left\|x+x^{\prime}\right\|_{1}}=\varphi_{\infty}^{m}(E, R) \leq \rho_{\infty}^{m}(E, R) \leq \psi^{m}(E, R)=\min _{x \notin P(E, R)} \max _{x^{\prime} \in P(x, E, R)} \frac{\gamma\left(x, x^{\prime}\right)}{\left\|x-x^{\prime}\right\|_{1}} .
$$

This implies the following statement that indicates the attainability of the bounds (2) in the case $p=\infty$.

Corollary 2. If for any pair of portfolios $x \notin P(E, R)$ and $x^{\prime} \in P(x, E, R)$ the equality $\left\|x+x^{\prime}\right\|_{1}=\left\|x-x^{\prime}\right\|_{1}$ holds, then the following formula is true:

$$
\rho_{\infty}^{m}(E, R)=\varphi_{\infty}^{m}(E, R)=\psi^{m}(E, R) .
$$

Corollary 3. For $m \in \mathbb{N}$, the stability radius $\rho_{1}^{m}(E, R)$ has the following lower and upper bounds

$$
\min _{x \notin P(E, R)} \max _{x^{\prime} \in P(x, E, R)} \gamma\left(x, x^{\prime}\right)=\varphi_{1}^{m}(E, R) \leq \rho_{1}^{m}(E, R) \leq m n \psi^{m}(E, R) .
$$

Now let us consider a numerical example that illustrates the attainability of the lower bound in Corollary 3.

Example 1. Let $m=2, n=3, X=\left\{x^{0}, x^{*}\right\}, x^{0}=(0,1,1)^{T}, x^{*}=(1,1,0)^{T}$, the pair of matrices $(E, R) \in \mathbb{R}^{2 \times 3} \times \mathbb{R}^{2 \times 3}$ :

$$
E=\left(\begin{array}{lll}
2 & 1 & 5 \\
4 & 0 & 2
\end{array}\right), R=\left(\begin{array}{lll}
6 & 2 & 3 \\
4 & 2 & 5
\end{array}\right)
$$

Then $\quad e\left(x^{0}, E\right)=6, \quad e\left(x^{*}, E\right)=4, \quad r\left(x^{0}, R\right)=7, \quad r\left(x^{*}, R\right)=8$. Hence $\quad x^{*} \notin P(E, R)$, $\left\{x^{0}\right\}=P\left(x^{*}, E, R\right)$. Therefore, $\gamma\left(x^{*}, x^{0}\right)=\min \{2,1\}=1, \varphi=\varphi_{1}^{2}(E, R)=1$. Next, we show that the stability radius $\rho_{1}^{2}(E, R) \leq \varphi=1$.

We define the pair of perturbing matrices $\left(E^{0}, R^{0}\right)$ as follows

$$
E^{0}=\left(\begin{array}{lll}
0 & 0 & 0 \\
\delta & 0 & 0
\end{array}\right), R^{0}=\left(\begin{array}{ccc}
-\delta & 0 & 0 \\
0 & 0 & 0
\end{array}\right),
$$

where $\delta>\varphi=1$. Then, taking into account $l=1$, it is easy to see that

$$
\begin{gathered}
e\left(x^{0}, E+E^{0}\right)-e\left(x^{*}, E+E^{0}\right)=2-\delta, \\
r\left(x^{*}, R+R^{0}\right)-r\left(x^{0}, R+R^{0}\right)=1-\delta .
\end{gathered}
$$


Therefore, $x^{*} \in P\left(E+E^{0}, R+R^{0}\right)$. From this and from the relations $\left\|E^{0}\right\|_{1}=\delta>1, \quad\left\|R^{0}\right\|_{1}=\delta>1$ and $x^{*} \notin P(E, R)$, it follows that $\rho_{1}^{2}(E, R) \leq 1$. Hence, according to Theorem 1 , we conclude $\rho_{1}^{2}(E, R)=\varphi_{1}^{2}(E, R)=1$.

Now let us show, that the upper bound $m n \psi^{m}(E, R)$ (see Corollary 3 ) is attainable for $m=1$ in the scalar case, i.e. when we choose a portfolio using only one linear criterion, for example, using only the criterion of the portfolio efficiency:

$$
\text { MINSUM ex }=\sum_{j \in N_{n}} e_{j} x_{j} \rightarrow \max _{x \in X},
$$

where $e=\left(e_{1}, e_{2}, \ldots, e_{n}\right) \in \mathbb{R}^{n}$. This problem requires finding the set of optimal portfolios $P(e)$. We thus consider an example with a particular class of the problems (7) where

Here

$$
\rho_{1}^{1}(e)=n \psi^{1}(e)
$$

$$
\psi^{1}(e)=\min _{x \notin P(e)} \max _{x^{\prime} \in P(x, e)} \frac{e\left(x-x^{\prime}\right)}{\left\|x-x^{\prime}\right\|_{1}} .
$$

Example 2. Let $p=1, X=\left\{x^{*}, x^{1}, x^{2}, \ldots, x^{n}\right\} \subset \mathrm{E}^{n}, n \geq 2$, where $x^{*}=(0,0, \ldots, 0)^{T} \in \mathbb{R}^{n}$, $x^{j}$ is the $j$-th column of the $\mathrm{n}$ identity matrix of size $n \times n$. Let $e=(a, a, \ldots, a) \in \mathbb{R}^{n}$, where $a>0$. Therefore, we have $e x^{*}=0, e x^{j}=a$, $j \in N_{n}$, i.e. $x^{*} \notin P(e), \quad x^{j} \in P(e)=P\left(x^{*}, e\right), \quad j \in N_{n}$. Hence, according to the definition of $\psi^{1}(e)$, equality $\psi=\psi^{1}(e)=a$ holds.

Now let $e^{\prime}=\left(e_{1}^{\prime}, e_{2}^{\prime}, \ldots, e_{n}^{\prime}\right)$ be a perturbing row vector from the row set $\Omega(n a)$, i.e. $\left\|e^{\prime}\right\|_{1}=\sum_{j \in N_{n}}\left|e_{j}^{\prime}\right|<n a$. It is easy to prove by contradiction that there exists an index $p$, such that $\left|e_{p}^{\prime}\right|<a$. Therefore, we derive

$$
\left(e+e^{\prime}\right)\left(x^{p}-x^{*}\right)=a+e_{p}^{\prime}>0 .
$$

Hence, we conclude that for any perturbing row $e^{\prime} \in \Omega(n \varphi)$ the portfolio $x^{*} \notin P\left(e+e^{\prime}\right)$. Therefore, since $x^{*} \notin P(e)$ we get $\rho_{1}^{1}(e) \geq n \psi^{1}(e)$. Thus, according to Corollary 3 , equality ( 8 ) is true.

Obviously, an example of a similar scalar problem involving portfolio risk minimization can be given when the stability radius is equal to the upper bound, as specified in Corollary 3. 


\section{Conclusion}

The obtained bounds for the stability radii of the problem at hand provide information on limit levels of changes in the initial data when all Pareto optimal solutions retain their required optimality properties. This information allows investors to make decisions and investments that are more financially robust, while incorporating possible uncertainties in risk and efficiency calculations.

\section{References}

[1] Best, M. J., Grauer, R. R., (1991). Sensitivity analysis for mean-variance portfolio Problems. Management Science, 37(8), 980-989. doi:10.1287/mnsc.37.8.980.

[2] Bronshtein, E. M., Kachkaeva, M. M., Tulupova, E.V. (2011). Control of investment portfolio based on complex quantile risk measures. J. of Comput. and Syst. Sci. Int., 50, 174-180. doi:10.1134/s1064230711010084.

[3] Chakravarti, N., Wagelmans, A. P. M. (1998). Calculation of stability radii for combinatorial optimization problem. Oper. Res. Lett., 23, 1-7. doi:10.1016/s01676377(98)00031-5.

[4] Christoffersen, P. F. (2003). Elements of Financial Risk Management. San Diego: Academic Press.

[5] Crouhy, M., Galai, D., Mark, R. (2005). The Essentials of Risk Management, New York: McGraw-Hill.

[6] Emelichev, V. A., Girlich, E., Nikulin, Yu. V., Podkopaev, D. P. (2002). Stability and regularization of vector problems of integer linear programming. Optim., 51(4), 645-676. doi:10.1080/0233193021000030760.

[7] Emelichev, V. A., Korotkov, V. V. (2012). On stability of a vector Boolean investment problem with Wald's criteria. Discrete Math. Appl., 22(4), 367-381. doi:10.1515/dma-2012-024,

[8] Emelichev, V., Korotkov, V. (2011). On stability radius of the multicriteria variant of Markowitz's investment portfolio problem. Bulletin of the Academy of Sciences of Moldova. Mathematics, 1, 83-94.

[9] Emelichev, V. A., Korotkov, V. V. (2013). Postoptimal analysis of bicriteria boolean problems of selecting investment projects with WaldB's and Savage's criteria. J. of Comput. and Syst. Sci. Int., 52(4), 608-617. doi:10.1134/s1064230713040072.

[10] Emelichev, V. A., Korotkov, V. V. (2012). Stability radius of a vector investment problem with Savage's minimax risk criteria. Cybernetics and Systems Analysis, 48(3), 378-386. doi:10.1007/s10559-012-9417-8.

[11] Guigues, V. (2011). Sensitivity analysis and calibration of the covariance matrix for stable portfolio selection. Computational Optimization and Applications, 48(3), 553579. doi:10.1007/s10589-009-9260-7.

[12] Hirschberger, M., Steuer, R. E., Utz, S., Wimmer, M., Qi, Y. (2013). Computing the nondominated surface in tri-criterion portfolio selection. Oper. Res. 61, 169-183. doi:10.1287/opre.1120.1140. 
[13] Van Hoesel, S., Wagelmans, A. (1999). On the complexity of postoptimality analysis of 0-1 programs. Discret. Appl. Math., 91, 251-263. doi:10.1016/s0166$218 \mathrm{x}(98) 00151-6$.

[14] Markowitz, H. M. (1991). Portfolio Selection: Efficient Diversification of Investments. New Yourk: Willey.

[15] Salo, A., Keisler, J., Morton, A. (Eds.) (2011). Portfolio Decision Analysis: Improved Methods for Resource Allocation (International Series in Operations Research and Management Science). New York: Springer.

[16] Savage, L. J. (1972). The Foundations of Statistics. New York: Dover Publ.

[17] Sotskov, Yu. N., Tanaev, V. S., Werner, F. (1998). Stability radius of an optimal schedule: A survey and recent developments. Industrial Applications of Combinatorial Optimization, Boston: Kluwer, 72-108. doi:10.1007/978-1-4757-28767 .4.

[18] Tapiero, C. S. (2010). Risk Finance and Asset Pricing: Value, Measurements, and Markets. New Yourk: Wiley. 